\title{
COMPARATIVE STUDY OF COLLAGEN AND GELATIN COATINGS ON TITANIUM SURFACES
}

\author{
Vanderleyden E. ${ }^{1}$, Van Hoorebeke L. ${ }^{2}$, Schacht E. ${ }^{1}$, Dubruel P. \\ 1. Polymer Chemistry \& Biomaterials Research group, Department of Organic Chemistry, University of Ghent, \\ Krijgslaan 281, 9000 Ghent, Belgium \\ 2. Linear Accelerator Facility, Department of Subatomic and Radiation Physics Chemistry, University of Ghent, \\ Proefstuinstraat 86, 9000 Ghent, Belgium
}

To date, critical bone defects are mainly treated by implantation of a bio-inert metal such as titanium (Ti) or nickel-titanium alloys. The surface of such biomaterials is in direct contact with the host tissue and thus plays a critical role in determining the biocompatibility. There are several reports indicating that osteointegration of these implants is not optimal. In order to improve the integration of implants, it is desirable to control interfacial reactions such that tissue-healing phenomena can be controlled. ${ }^{1}$ Our goal is to apply natural polymers as stable bio-active coatings for porous Ti scaffolds.

To achieve such a stable polymeric coating, we have applied a four-step-procedure: (1) cleaning, (2) oxidation and (3) silanisation of the titanium surface followed by (4) polymer immobilisation (figure 1). In the present work we compared the immobilisation of collagen type I with the immobilisation of its hydrolysed derivative, gelatin. Collagen is one of the most usefull biomaterials due to its excellent biocompatibility, biodegradability and weak antigenicity. Still, a more economical choice would be gelatin.

Collagen was immobilised onto the Ti surface in an adsorptive and in a covalent manner. ${ }^{2,3}$ Only the latter proved to lead to a stable coating. This method comprised of a silanisation with 3aminopropyltriethoxysilane (APTES) to introduce amines to the Ti surface. In a next step, these amines can react with the carboxylic acid groups of collagen by using a coupling reagent. Without any doubt, there will also be intra- and intermolecular crosslinking between the collagen chains. In case of gelatin, we followed a different approach. Gelatin was modified with metacrylamide groups (Gel-mod) and the $\mathrm{Ti}$ surface was modified with methacrylate groups through silanisation using 3(trimethoxysilyl)propyl methacrylate (TMSPMA). Crosslinking between both was achieved by e-beam treatment.

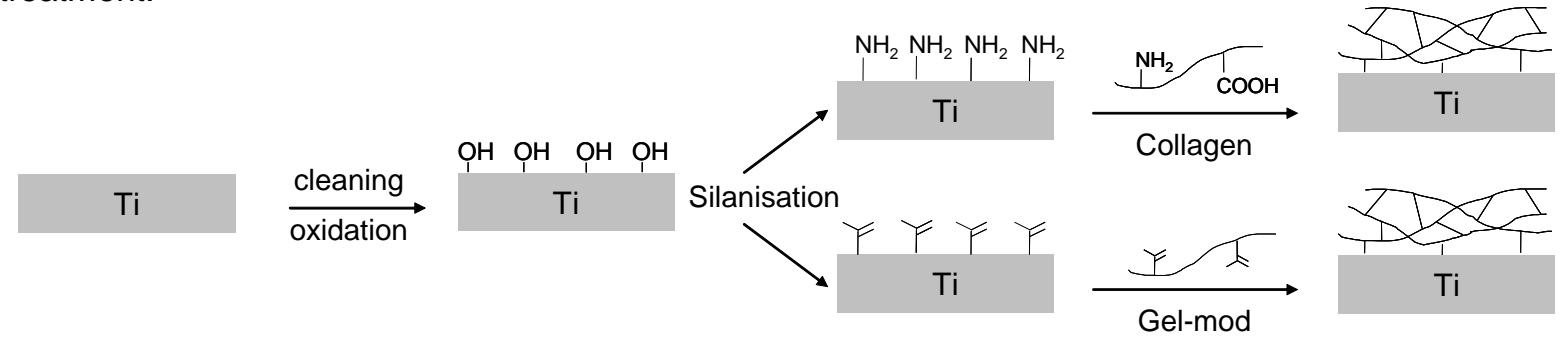

Figure 1. Schematic overview of the immobilisation strategies of collagen and gelatin.

\section{$\underline{\text { References: }}$}

1) Nanci, A., Wuest, J.D., Peru, P. et al. (1998). J Biomed Mater Res, 40, 324-335

2) Dupont-Gillain C.C., Jacquemart I., Rouxhet P.G. (2005). Colloids and Surfaces B: Biointerfaces, 43, 179-186

3) Müller R., Abke J., Schnell E. et al. (2006). Biomaterials, 27, 4059-4068

The authors acknowledge the financial support of the Institute for the Promotion of Innovation by Science and Technology in Flanders (Belgium), the IWT-SBO project GBE with KUL, VITO and UGent as partners and the Belgian Research Policy (IUAP-V-03). 